\title{
Residual stresses of types II and III and their estimation
}

\author{
P KESAVAN NAIR and R VASUDEVAN \\ Department of Metallurgical Engineering, Indian Institute of Technology, \\ Madras 600036, India
}

\begin{abstract}
Residual stresses (RS) are induced in metallic materials by a variety of working and fabrication processes. They are generally classified into three categories (types I, II and III) depending upon their range of influence. Separation of type I and II stresses often requires stress measurements on thin specimens. Type III stresses are related closely to grain fragmentation and micro-strains associated with plastic deformation. Perhaps the oldest and the most rigorous method of estimating these stresses is still the Warren-Averbach analysis developed during the late forties/early fifties. Other techniques involving integral breadths and variance of the profiles have also been developed. In all these methods developed during the early stages, prior to seventies, a major requirement was well-separated non-overlapping profiles. The late sixties and early seventies saw a dramatic increase in computational capabilities with the advent of powerful electronic computers. This led to the introduction of curve-fitting procedures into the field of $\mathrm{X}$-ray diffraction. The most remarkable achievement of this period is the development of the Rietveld Method. Although this method was initially developed to tackle the neutron diffraction profiles, which are as a rule nearly symmetric and Gaussian in nature, the method saw rapid developments during the eighties. At present, techniques based on concepts developed by Rietveld could be applied to essentially asymmetric and non-Gaussian multiple spectral components of X-ray diffraction profiles. Pattern decomposition techniques which separate composite powder diffraction profiles into individual profiles are now available. In combination with single line profile analysis techniques, this provides a powerful tool in the hands of researchers. A typical example of such a single line profile analysis is given.
\end{abstract}

Keywords. Residual stresses; microstresses; microstrain; line profile analysis; profile fitting.

\section{Introduction}

Residual stresses present in a material can be broadly classified into two major categories: macro- and micro-residual stresses. The macro residual stresses are some times referred to as stresses of type I. Following the latter scheme, the micro-residual 


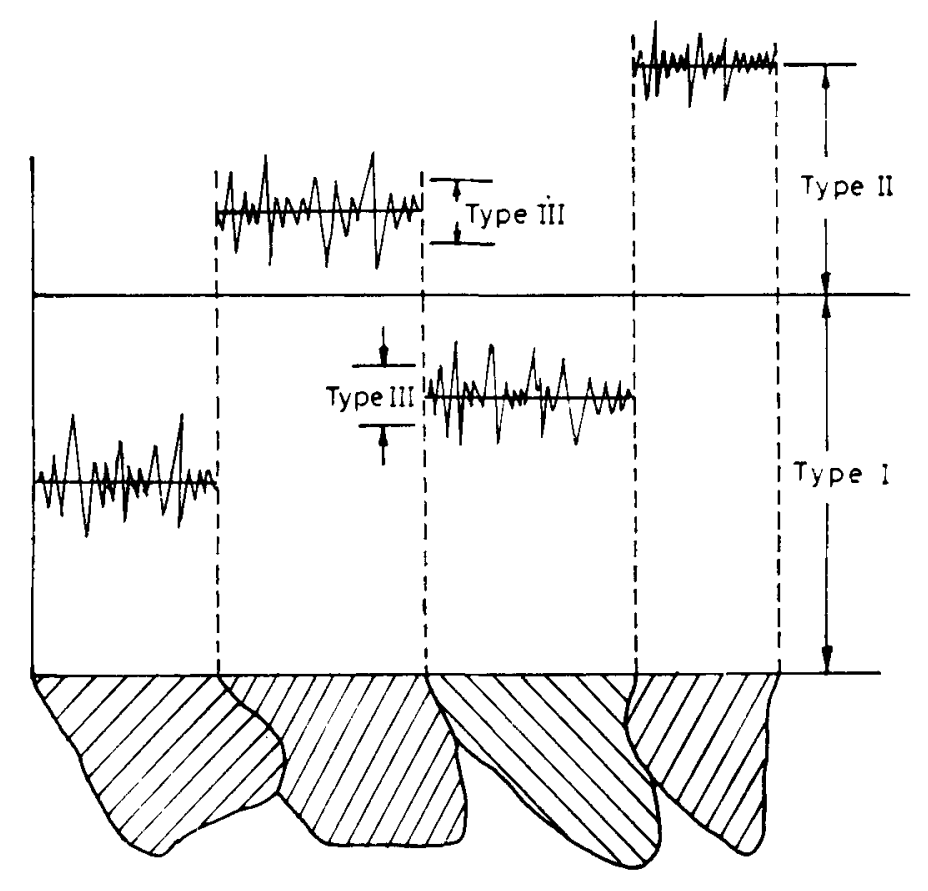

Figure 1. Schematic representation of residual stresses of types I, II and III.

stresses are further classified into two: stresses of types II and III. The basis of this classification is shown in figure 1 . The evaluation of type I or macro residual stresses are by now well established (Macherauch et al 1973; Hauk \& Macherauch 1984). This paper gives an introduction to the more widely used methods for the evaluation of stresses of types II and III. Although both these stresses are commonly referred to as micro-residual stresses, the estimation of these two types of stresses follows different principles. While the evaluation of type III stresses has achieved a relatively high degree of sophistication, that of type II stresses is still relatively difficult; accurate and reliable methods comparable to those available for the evaluation of the other two types are still to be evolved. The principle of X-ray evaluation of type 1 stresses is based on an accurate measurement of interplanar spacings of a particular set of crystallographic planes. Since both types I and II stresses affect this spacings in a similar manner, any attempt to evaluate the former without taking into account the effect of the latter is bound to be inaccurate. The estimation of type II stresses is discussed in this specific context.

\section{Evaluation of type II stresses}

In general, all the three types of stresses are likely to be present in a polycrystalline bulk sample. Stresses of types II \& III will directly affect the lattice strains. A possible definition of type II stresses could be that it is those stresses existing in the crystals even after complete removal of type I stresses. Indeed, strictly speaking, type II stresses will very greatly from grain to grain. In a tine-grained polycrystalline material it becomes 
rather difficult to evaluate such stresses on a grain-to-grain basis. This necessarily means that only an average value can be obtained. The method of measurement is the susal multiple exposure X-ray diffraction (XRD) technique. Since both type I and type II stresses affect the diffraction profile in a similar fashion, the results obtained through a routine residual stress measurement through the multiple exposure technique are, in general, a sum of these two stresses. The proportion in which they contribute to the total can vary greatly.

Type II stresses can be defined in a more precise way as

where

$$
\sigma_{I I}=[\sigma \mathrm{d} a / \mathrm{d} a] \text { one crystallite }-\sigma_{I}
$$

$$
\sigma=\sigma_{I}+\sigma_{I I}+\sigma_{I I I}
$$

and the subscripts I, II, and III means the stresses of types I, II, and III.

The separation of type I and type II stresses is possible only if the distribution is of a specific type, in specimens conforming to definite geometrical requirements (Hauk \& Nkolin 1988): for example, in strips and sheets, if the distribution is symmetrical, it is possible to estimate the distribution across the depth. This stipulation arises from the fact that individual estimation of type I or type II stresses can be done only through deflection methods. As is well known, the principle behind the estimation of residual stresses through deflection methods depends upon relating the dimensional or shape changes of a specimen caused by removal of material to the relieved stresses. This in turn requires that the geometry of the specimen be simple (Treuting \& Read 1951).

Taking the case of thick strips with a symmetrical stress distribution of type I and type II stresses, a procedure having the following steps can be adopted: (1) Successive layers are removed from both top and bottom surfaces of the specimen in a symmetrical fashion, which ensures that the removal as such will not have any significant effect on the subsequent measurements. Consequently, a routine measurement employing a multiple exposure technique on the surface layers after symmetrical etching can provide type I + type II stress distributions as functions of depth in this type of specimen. (2) A straightforward stress estimation employing the deflection methods can provide the distribution of type I stresses alone. In this case the removal of material is only from one side. The deflection produced can be related to the stresses of type I present in the material. (3) Thin specimens are carefully removed from various positions of the sample in such a way that the removed thin slice is essentially free of type I stresses (Hauk 1983). A routine residual stress measurement employing multiple exposure technique can provide the distribution of type II stresses. The essential features of the above three steps are illustrated in figure 2. If step 3 could be successfully completed, a direct evaluation of type II stresses is obtained, which can be cross checked employing the additive nature of type I and type II stresses obtained in steps 1 and 2. However, it is to be remembered that steps such as the one just detailed are rather specific in application. A general method to evaluate the type II stress distribution is not yet available.

It is clear from the above discussions that the estimation of type II stresses involves residual stress measurement employing multiple exposure X-ray diffraction techniques. All the limitations applicable to the evaluation of residual stresses employing this technique such as linearity of the $D$ vs $\sin ^{2} \Psi$ plots, existence of sharp stress gradients normal to the surface, coarse grain size etc can cause problems in these cases as well (Hauk 1986). 

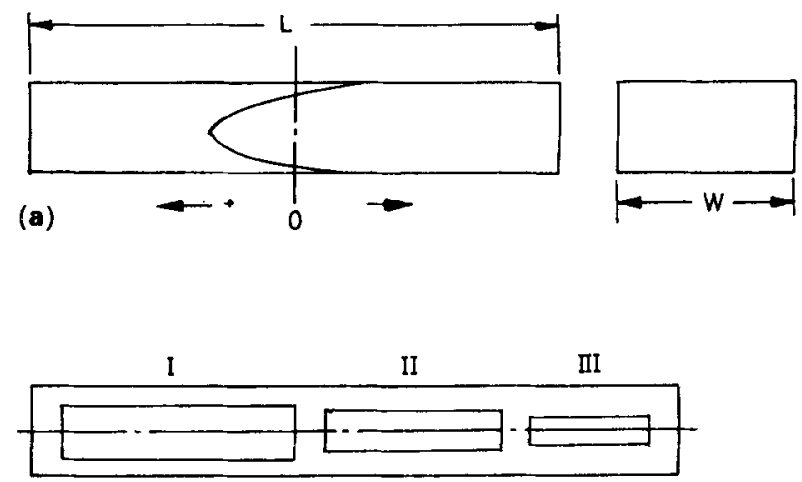

(b)

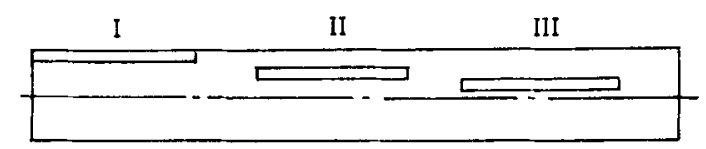

(c)

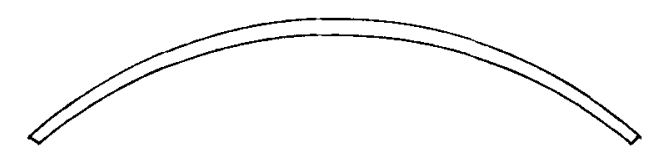

(d)
Figure 2. Evaluation of various types of stresses on samples with symmetrical distribution of residual stresses across the thickness, (a) Geometry of specimen with residual stress distribution. (b) Location of specimen for evaluation of $\sigma_{I}+\sigma_{I I}$ after symmetrical etching. (c) location of specimen for evaluation of II. (d) Deflection of specimen on etching on one side for estimation of $\sigma_{I}$.

\section{Type III stresses and their evaluation}

Type III stresses, as illustrated in figure 1 , have a range of action relatively small when compared to the other two. As a consequence, they are estimated as average values. Their effect on the diffraction profile is also different. If present, they tend to change the shape of the profile, rather than the position. Unfortunately, there are quite a number of factors which can affect the shape of the diffraction profile: the dimensions and morphology of the coherently diffracting domains, nonstochiometry, faults dislocations, instrument geometry etc., apart from internal stresses. This list is only illustrative and not exhaustive. Although a number of parameters are required to characterize the shapes of diffraction profiles, the one most widely used is the breadth of the line profile.

It is generally accepted that the observed diffraction profile (the $h(2 \theta)$ profile) is a convolution of the instrument aberration $(g(2 \theta)$ profile) and the pure diffraction profile which is due to the specific micro freatures of the sample $(f(2 \theta)$ profile) (Wilson 1963; Warren 1969). The can be written as

$$
h(2 \theta)=g(2 \theta) \times f(2 \theta)=g\left(2 \theta^{\prime}\right) f\left(2 \theta-2 \theta^{\prime}\right) d(2 \theta) .
$$


Since each point in the observed profile is assumed to be the result of a summation of the product of $g$ and $f$ over all possible values of $f(2 \theta)$, the computations involved are quite time consuming. One option would be to do this computation in Fourier space; alternately it can be done analytically, rather than numerically, through a proper choice of functions to present the individual profiles. Chronologically, the Fourier methods found wide acceptance, much before the second option was implemented.

The components contributing to the diffraction profile as mentioned above are discussed individually below.

\subsection{The pure diffraction profile $f$}

The principal contributors to this profile are the 'particle size' or the coherently diffracting domain size. This size $D$ is related to the breadth of the size profile through the relation named after Scherrer:

$$
\beta(D)=k \lambda / D \cos \theta
$$

where $\theta$ is the Bragg angle, and $k$ a constant close to 1 .

The second contributing factor to the $f$ profile is the microstrain or the residual stresses of type III. This also broadens the residual stress profile according to the relation:

$$
\beta(e)=4 \in \tan \theta .
$$

It is generally believed that both these profiles are symmetrical in shape. The former due to particle size is assumed to be having a Cauchy (Lorentzian) and the latter a Gaussian profile (Halder \& Wagner 1966; Nandi \& Sengupta 1978; de Keijser et al 1982).

It is to be remembered that other factors such as compositional inhomogeneities might also affect the breadths of this profile. But generally such effects are assumed to be negligible.

Although the term particle size is used to refer to the coherently diffracting domain size, the terminology is valid perhaps only in the case of inorganic materials where strain effects are negligible. The apparent domain size derived from the breadth of the diffraction profile is a mean length normal to the diffracting planes, averaged over the entire irradiated diffracting volume of the sample. It is to be remembered that size broadening depends strongly on the shape or form of the domain. Consequently the breadth of the profile is likely to be anisotropic in the sense that there might be considerable variations depending upon the reflections considered.

The breadth usually could be the full width at half maximum (FWHM) or the integral breadth (IB). While the former is not amenable to any direct physical interpretation, the latter, in reciprocal space, can be thought of as the volume weighted average crystalline size i.e.

$$
D=\beta^{*}=\beta \cos \theta / \lambda,
$$

where $\beta$ is the integral breadth on a $2 \theta$ scale and $\beta^{*}$ is the integral breadth on a $d^{*}$ (i.e. $\sin \theta / \lambda)$ scale.

\subsection{The strain broadening}

As mentioned before, the broadening due to the lattice strain is considerd to be nearer to Gaussian in shape. However due to anisotropy of elastic properties, the breadths 
need not vary smoothly with Bragg angle (Delhez et al 1980). The breadth will thus very much depend upon the particular reflection considered.

\subsection{The instrumental profile $g$}

The instrumental profiles vary smoothly with angle (Cox et al 1988). The profile shape may also vary between pure Gaussian and pure Cauchy. At low angles, geometric aberrations are predominant and at high angles, effects due to wavelength distributions predominate. Usually the $g$ profile is experimentally obtained as the profile from a well-annealed defect-free standard sample.

\subsection{Separation of size and strain effects}

3.4a Methods based on integral breadths: Classical or conventional ways of separation require at least two orders of the same reflection in order to separate the size and strain effects. However, before attempting such a separation it is essential that the instrumental effects are eliminated from the observed profile. For this purpose the experimentally obtained $g$ profile from an appropriate standard material is used. As a first step the effect due to the doublets is eliminated through analytical procedures such as the Rachinger's method (Pease 1948; Rachinger 1948) and the pure diffraction profile $f$ is obtained through any of the following relationships (Jagodzinski 1949; Anantharaman \& Christian 1956; Taylor 1961),

$$
\begin{aligned}
\beta & =B-b \\
\beta & =B-b^{2} / B \\
\beta^{2} & =B^{2}-b^{2}
\end{aligned}
$$

where $\beta$ is the corrected breadth, $B$ and $b$ are the breadths of observed and instrumental profiles after eliminating the wavelength dispersion effects. Equation (7) assumes that both the observed profiles are Lorentzian and (9) assumes that both are Gaussian in shape. Equation (8) yields a value in between. This corrected breadth $\beta$ includes the effects due to both size and strain effects.

The procedure for obtaining the strain or particle size from this corrected breadth involves the assumption that the breadth of the size profile $\beta_{D}$ and that of the strain profile $\beta_{e}$ are related through (Anantharaman 1959; Rama Rao \& Anantharaman 1963)

$$
\beta^{2}=\beta_{D}^{2}+\beta_{e}^{2}
$$

This when combined with (4) and (5) above yields a linear relationship between $(\beta \cos \theta / \lambda)^{2}$ and $(\sin \theta / \lambda)^{2}$ with a slope of $16 \epsilon^{2}$ and an intercept of the reciprocal of the square of the apparent crystallite size. It is the necessity of plotting (10) to obtain the slope and intercept, which dictates that at least two orders of reflections be available. Alternately, if the sample is reasonably isotropic, one can use the breadths of other reflections as well for the above plot. The size obtained in this manner includes effects due to stacking faults, dislocations etc.

Yet another method of obtaining the pure diffraction profile in a more direct fashion is the well-known Stoke's correction (Stokes 1948; Warren 1969). Here the removal of effects due to the instrumental profile is carried out in Fourier space by expressing the 
observed and the experimentally obtained $g$ profile as a Fourier series. The Fourier coefficient of $f$ profile can be shown to be given by

$$
F(n)=H(n) / G(n),
$$

where $n$ is the harmonic number and $F(n), G(n)$ and $H(n)$ are the respective Fourier coefficients of $f, g$ and $h$ profiles. This method is completely rigorous and makes no special assumptions regarding the profile shape. Once the coefficients of the pure diffraction profile are obtained, the latter can be synthesized and treated in a similar fashion as above. However, a more elegant way of separating the strain and size effects is the Warren-Averbach Method, discussed below (Warren \& Averbach 1950, 1952).

3.4b The Warren-Averbach method: The Fourier real coefficients of the pure diffraction profile can be related to the coefficients of the size and strain profiles through

$$
A(L)=A^{D}(L) A^{\epsilon}(L)
$$

where $L$ refers to a distance normal to the ciiffracting planes and the superscripts $D$ and $\epsilon$ to particle size and strain respectively. For small values of $L$, the coefficients of the size and strain profiles can be approximated by

$$
\begin{aligned}
& A^{D}(L)=1-L / D_{h k l}, \\
& A^{\epsilon}(L)=1-2 \pi^{2} L^{2}\left[\left\langle\epsilon^{2}\right\rangle-\langle\epsilon\rangle^{2}\right] S_{0}^{2},
\end{aligned}
$$

where $D_{h k l}$ is the domain size normal to the reflecting planes $h k l$ and $\in$ is the microstrain or type III strain. $S_{0}^{2}$ is given by

$$
S_{0}^{2}=(2 \sin \theta / \lambda)^{2}=\left(h^{2}+k^{2}+l^{2}\right) / a^{2} .
$$

Combining (12)-(14) for small values of $L$, one obtains

$$
\ln A(L)=\ln A^{D}(L)-2 \pi^{2} L^{2}\left[\left\langle\epsilon^{2}\right\rangle-\langle\epsilon\rangle^{2}\right] S_{0}^{2} .
$$

Thus a plot of $\ln A(L)$ against $S_{0}^{2}$ will be a linear one with the intercept giving $\ln A^{D}(L)$, the particle size coefficient. The slope of this plot is a measure of the microstrain, given by $\left[\left\langle\epsilon^{2}\right\rangle-\langle\epsilon\rangle^{2}\right]$ :

$$
\begin{aligned}
& \left\langle\epsilon_{L}^{2}\right\rangle^{1 / 2}=\left[\left\langle\epsilon^{2}\right\rangle-\langle\epsilon\rangle^{2}\right]^{1 / 2} \\
& =\left[\ln A^{D}(L)-\ln A(L)\right]^{1 / 2} /(2 \pi) L S_{0},
\end{aligned}
$$

where $\epsilon_{L}$ is the component of the strain normal to the reflection plane, averaged over the distance $L$. This strain is a function of the averaging distance and usually decreases with $L$, reaching an asymptotic value for large $L$. An accurate and acceptable interpretation of this plot is still not available.

\subsection{Limitations on the application of integral breadth and Fourier methods}

Both integral methods and Fourier methods presume the existence of well separated and independent reflections from the sample. All too often situations arise where the peaks are extensively overlapped by neighbouring reflections. Very often even moderately well-resolved reflections might be absent. Such complex patterns are very common in modern materials such as composites, ceramics, polymers etc. The reflections may rise 
not only from well crystallized grains, but also from quasicrystalline, nanocrystalline or amorphous materials. Analysis of such patterns are becoming a necessity in the present day context. Fortunately, pattern decomposition methods are available now which enable one to handle such situations with a reasonable degree of confidence.

\subsection{Methods based on profile refinement}

As mentioned above, most of the classical methods require well-resolved powder diffraction profiles and input data of high quality. These two requirements often lead to a dead end. The entire process of profile analysis got new life and interest in it was renewed with the introduction of least squares structure refinement based on concepts originally proposed by Rietveld $(1967,1969)$. The basic idea was to least square refine the parameters until the best fit is obtained between the entire observed diffraction pattern as a whole and a calculated pattern based on simultaneously refined parameters which affect the diffractogram one way or the other. Factors Such as crystal structure, instrumental effects, specimen imperfections or any other effect could be included so long as they can be modelled. An important feature of this method is the feedback during the refinement process itself between the improving goodness of fit and the relevant structural parameters. What is refined are the parameters themselves and not the profile. Although the method was originally proposed for the analysis of neutron diffraction profiles, it was quickly realized that its domain of application is really vast. In the present context the method has high potential to provide data on the structural imperfections of the material to be analysed.

An essential requirement for this purpose is to ascribe a suitable analytical function to the line profile. Early workers, as already mentioned, assumed a wholly Gaussian or a Lorentzian profile. However, later it was shown that deficiencies do exist in both these cases (Suortti et al 1979; Young 1980). At present it is recognized that a pseudo-Voigt or a Pearson VII profile shape function can be considered to be most suitable for the purpose (Hall et al 1977; Young et al 1982; Thompson et al 1987). The Voigt function is the result of an analytical convolution of a Gaussian and a Lorentzian,

$$
V(x)=I_{0}\left[\beta / \beta_{c} \beta_{g}\right] \int C^{V}(z) G^{V}(x-z) \mathrm{d} z
$$

with

and

$$
C^{V}(x)=\left[1+\left(\pi^{2} x^{2} / \beta_{c}^{2}\right)^{-1}=\left(1+4 x^{2}\right) /\left(2 w_{c}\right)^{2}\right]^{-1}
$$

$$
G^{v}(x)=\exp \left(-\pi x^{2} / \beta_{G}^{2}\right)=\exp \left[(-4 \ln 2) x^{2} /\left(2 w_{G}\right)^{2}\right]
$$

where $C^{v}$ and $G^{v}$ indicate the Cauchy and Gaussian components having integral breadths of $\beta_{C}$ and $\beta_{G}$ respectively with $2 w_{C}$ and $2 w_{G}$ as the corresponding full widths at half maximum. $I_{0}$ is the peak maximum intensity (at $x=0$ ) and $\beta$ the integral breadth of the Voigt profile, $V(x)$. As can be seen, the range of profiles that can be handled varies from pure Gaussian to pure Cauchy, depending on the ratio of the component widths. The pseudo-Voigt function on the other hand provides for a mixing parameter in the form of fractions of superposed Lorentzian and Gaussian components needed to fit an observed profile (Hindeleh \& Johnson 1972),

$$
p V(x)=I_{0}\left[C^{p V}(x)+G^{p V}(x)\right]
$$


where

with

$$
\begin{aligned}
& C^{p V}(x)=\eta\left[1+\Pi^{2} x^{2} /\left(\beta_{c^{\prime}}\right)^{2}\right]^{-1}, \\
& G^{p V}(x)=(1-\eta) \exp \left[-\Pi^{2} x^{2} /\left(\beta_{G}\right)^{2}\right],
\end{aligned}
$$

$$
0 \leqslant \eta \leqslant 1 \text {. }
$$

The subscripts $C^{\prime}$ and $G^{\prime}$ denote that the respective integral breadths refer to Cauchy and Gaussian profiles respectively. $\eta$ is the fraction of the Cauchy component required to obtain the best fit during the least squares process. In most of the modern computer programs this fraction itself is one of the parameters to be refined during execution. Since the Gaussian is a relatively broad profile and the Lorentzian a narrow one, the mixing of the two can represent a symmetric profile. The Pearson VII is also a symmetric profile function which can equally well represent a symmetric diffraction profile:

$$
p V I I(x)=I_{0}\left(1+x^{2} / m a^{2}\right)^{-m}, 1 \leqslant m \leqslant \infty,
$$

with

$$
a=\beta \Gamma(m) /[m \pi)^{1 / 2} \Gamma(m-1 / 2),
$$

where $m=1$ implies a pure Cauchy profile and $m=\infty$, a pure Gaussian one. The Gamma Function is denoted by the symbol $\Gamma$. Both Voigt and Pearson VII functions can represent asymmetrical profiles by employing the respective split-type functions. In this case, the diffraction profile is split into a low angle side and a high angle side at the peak maximum. Two different Pearson VII or Voigt functions, as the case may be, are then fitted on either side. In other words the profile is represented by two functions having a common peak maximum position and intensity (Snyder 1993). However, they may have different values of full width at half maximum, decay rates, and other profile parameters. Further, the functions denoted by $P(x)_{\text {low }}$ and $P(x)_{\text {high }}$, representing the low and high angle sides of the profile should satisfy the following condition:

$$
\int_{-\infty}^{T} P(x)_{l o w} \mathrm{~d} x+\int_{T}^{+\infty} P(x)_{h i g h} \mathrm{~d} x=1 .
$$

In most of these refinement procedures a number of profile parameters like FWHM, the integrated intensity, the peak position etc. are simultaneously refined and final optimized values obtained. Once an optimized profile fit is obtained, the Gaussian and the Cauchy components of the profile can be separated, and the size and strain calculated based on (4) and (5) assuming the Cauchy breadth for size and the Gaussian breadth for strain (de Keijser et al 1982).

Another approach is to obtain the Cauchy and Gaussian integral breadths from the experimental values of the integral breadth $(\beta)$ and the ratio $(\phi)$ of the fullwidth at half maximum to the integral breadth of the $g$ and $h$ profiles. This can be done graphically (Langford et al 1988) or using empirical equations such as (de Keijser et al 1982),

$$
\begin{aligned}
& \beta_{C}=\beta\left(2.0207-0.4803 \phi-1.7756 \phi^{2}\right) \\
& \beta_{G}=\beta\left[0.6442+1.4187(\phi-2 / \pi)^{1 / 2}-2.2043 \phi+1.8706 \phi^{2}\right]
\end{aligned}
$$




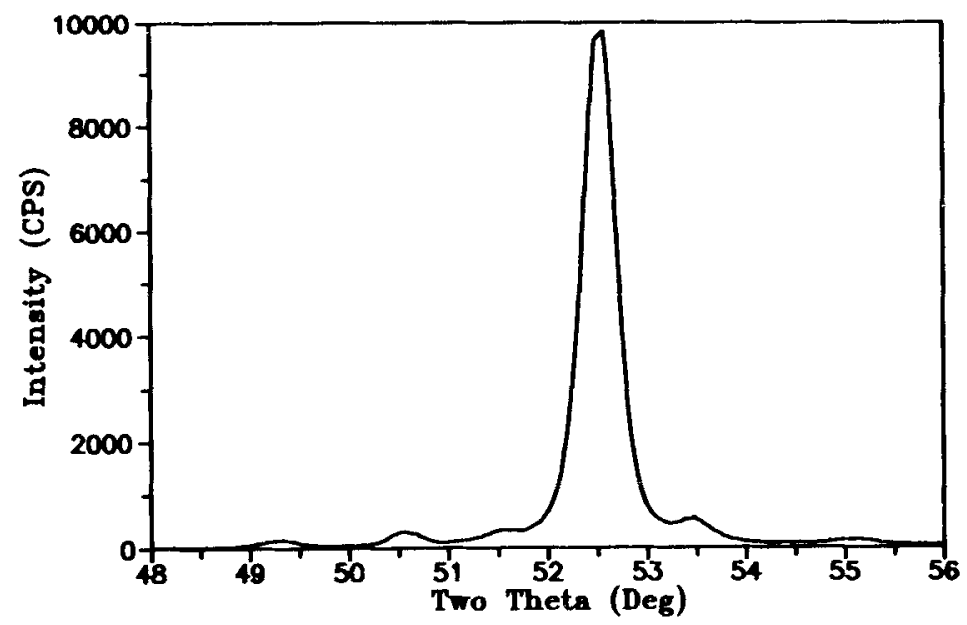

Figure 3. The experimental profile from electroless $\mathrm{Ni}-\mathrm{P}$ deposit with 4.4 wt. \% phosphorus after annealing at $400^{\circ} \mathrm{C}$ for $2 \mathrm{~h}$.

Once the Cauchy and Gaussian breadths of the experimental $g$ and $h$ profiles are known those of the $f$ profile can be calculated employing equations similar to (7) and (9). Further, the crystalline size and strain could be estimated using the Cauchy and Gaussian breadths of the $f$ profile in (6) and (5) respectively.

Alternately, if two orders of the reflection are available, the integral breadths of the total profile can be straightaway made use of to obtain the size and strain components.

A cross-comparison between the results of a multiple line analysis and single line analysis might not produce identical values for profile parameters. This is mainly

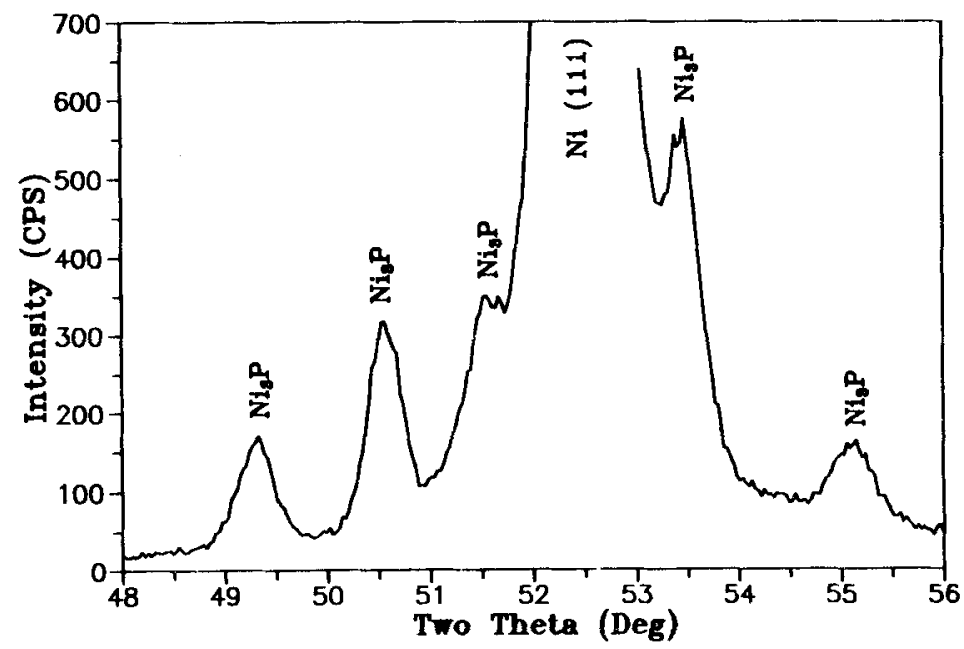

Figure 4. The experimental profile from electroless Ni-P deposit with $\mathbf{4 . 4}$ wt. \% phosphorus after annealing at $400^{\circ} \mathrm{C}$ for $2 \mathrm{~h}$ (enlarged intensity scale). 
because the weightage of the Gaussian and Cauchy contents for different orders of reflection will not, in general, be the same (de Keijser et al 1983).

A typical example of a single line size-strain analysis is given below in order to demonstrate the capabilities of the approach.

\subsection{Profile separation and single line profile analysis: An example}

In figure 3 an experimental profile obtained from a $\mathrm{Ni}-\mathrm{P}$ electroless deposit on mild steel substrate is presented. The diffractogram was obtained after annealing the sample for $2 \mathrm{~h}$ at $400^{\circ} \mathrm{C}$. The $\mathrm{P}$ content of the deposit was $4.4 \%$. As is evident there is extensive overlap between the main high intensity $\mathrm{Ni}(111)$ profile and the emerging $\mathrm{Ni}_{3} \mathrm{P}$ peaks. The same pattern is shown on an enlarged scale to have a better appreciation of the extensive overlap between the profiles in figure 4 . The profile was deconvoluted with a least square fitting procedure employing the pseudo Voigt function (Toraya 1986). The separated profiles are presented in figure 5. As mentioned earlier, a number of parameters are simultaneously refined. The final refined parameters are presented in table 1.

For the purpose of single line analysis, annealed pure $\mathrm{Ni}$ powder was used as a standard. The refined parameters obtained from this profile (not shown) were used for correcting the observed profile breadths and the size and strain calculated employing (4) and (5).

\section{Concluding remarks}

Procedures for separating type I and type II stresses are discussed. The separation procedure is a combination of $\mathrm{X}$-ray diffraction and mechanical dissection type of techniques. Consequently a complete distribution of type II stresses can be obtained

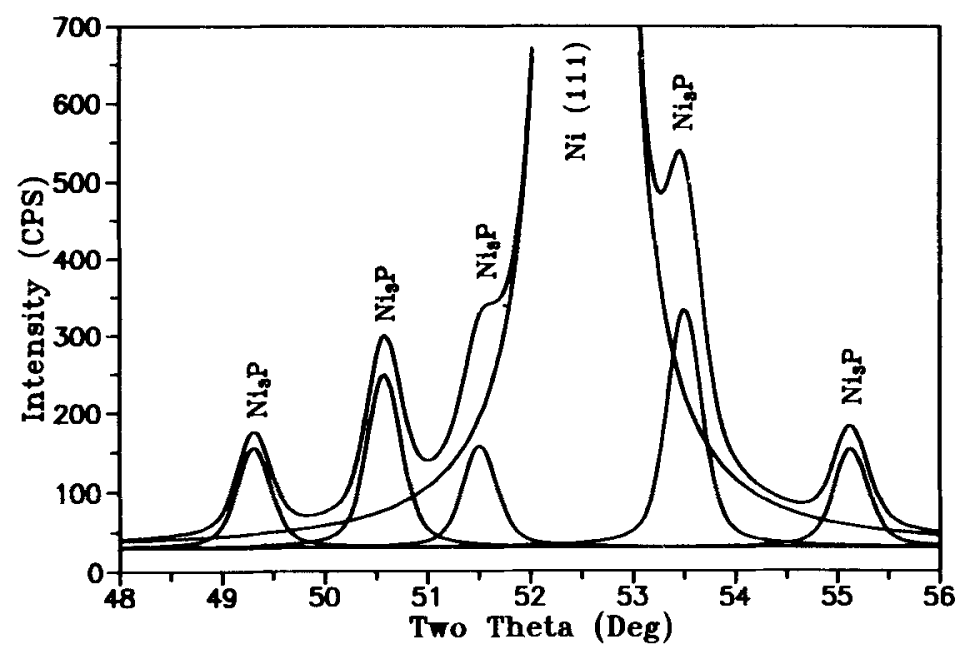

Figure 5. The deconvoluted individual diffraction profiles and total profile corresponding to figure 4 . 
Table 1. Parameters from profile fitting and results of single line analysis.

\section{Parameters from profile fitting}

Parameters are in degrees (Two theta scale)

Profile shape : pseudo-Voigt

Radiation used : cobalt

$L 1=1.788965, L 2=1.792850, K=0.4970$

Theta range : $48 \cdot 00-56.00$ Step : 0.03

$\mathrm{B} 1=30.03 \mathrm{~B} 2=0.00 \mathrm{~B} 3=0.00$

Profile identification: $\mathrm{Ni}(111)$

Integrated Intensity

Peak intensity $(A 1+A 2)$

$5179 \cdot 866000$

$10018 \cdot 730000$

52.529820

0.517018

0.122791

Integral breadth $(A 1+A 2)$

Alpha 1

Integrated intensity

Peak intensity

Peak maximum position

Integral breadth

FWHM

Cauchy content

Gaussian content

Cauchy breadth

Gaussian breadth
3453.244000

$7273 \cdot 720000$

52.504000

0.474756

0.357625

0.519539

0.480461

0.309947

0.249624
Alpha 2

1726.622000

3612.031000

52.626790

0.478020

0.357625

0.537562

0.462438

0.320140

0.244629

\section{Results of single line analysis}

Profile identification : Ni(111)

Integral breadth of diffraction profile $\quad=0.3875569$

FWHM of diffraction profile $=0.3038880$

Cauchy breadth of diffraction profile $\quad=0.1888990$

Gaussian breadth of diffraction profile $\quad=0.2057786$

Cauchy content of diffraction profile $\quad=0.5129336$

Gaussian content of diffraction profile $\quad=0.4870664$

Particle size $=605 \AA$

Micro strain $=1.821 E-03$

only under limited conditions. The estimation of type III stresses has made remarkable advance in recent years. Induction of the Rietveld concepts into profile analysis has given greater flexibility in their estimation.

\section{References}

Anantharaman T R 1959 Indian Institute of Science Golden Jubilee Research Volume, p. 280 Anantharaman T R, Christian J W 1956 The measurement of growth and deformation faulting in hexagonal cobalt. Acta Crystallogr. 9: 479 
Cox D E. Toby B H, Eddy M M 1988 Acquisition of powder diffraction data with synchrotron radiation. Aust. J. Phys. 41: 117-131

De Keijser Th H, Langford J I, Mittemeijer E J, Vogels A B P 1982 Use of Voigt function in a single line method for analysis of X-ray diffraction line broadening. J. Appl. Crystallogr. 15: $308-314$

De Keijser Th H, Mittemeijer E J, Rozentaal H C F 1983 The determination of crystallite size and lattice strain parameters in conjunction with the profile refinement method for the determination of crystal structures. J. Appl. Crystallogr. 16: 309-316

Delhez R, De Keijser Th H, Mittemeijer E J 1980 Accuracy on powder diffraction: Natl. Bur. Stand. Special Publication 567 (eds) S Block, C R Hubbard (Washington, DC: NBS) pp. $212-253$

Halder N C. Wagner C N J 1966 Analysis of broadening of powder pattern peaks using variance, integral breadth and fourier coefficient of line profile. Adv. X-ray Anal. 9:91-102

Hall Jr, Veeraraghavan M M, Rubinh V G, Winchell P G 1977 Approximation of symmetric X-ray peaks by Pearson Type-VII distribution. J. Appl. Crystallogr. 10: 66-68

Hauk V 1983 Residual stresses, their importance in science and technology (eds) E Macherauch, V Hauk (Oberursel: Deutsche Gesellschaît fuer Materialkunde (DGM)) pp. 1-9

Hauk V 1986 Residual stresses in science and technology. (eds) E Macherauch. V Hauk (Oberursel: Deutsche Gesellschaft fuer Materialkunde (DGM)) 1: 353361

Hauk V, Macherauch E 1984 A useful guide for X-ray stress evaluation. Adv, X-ray Anal. 27: 81.99

Hauk V, Nkolin H J 1988 Texture Microstruct. 8 \& 9: 693-716

Hindeleh A M, Johnson D J 1972 Crystallinity and crystallite size measurement in cellulose fibres: 1 Ramie and Fortisan. Polymer 11: 423430

Jagodzinski H 1949 Ein Dimensionale Fehlordnung in Kristallen und ihr Einfloss aus die Roentgeninterferen Zen-I. Acta Crystallogr. 2: 201

Langford J I. 1978 A rapid method for analysing the breadths of diffraction and spectral lines using the voigt function. J. Appl. Crystallogr. 11:10-14

Langford J I, Delhez R. De Keijser Th H. Mittemeijer E J 1988 Profile analysis for micro crystalline properties by the fourier and other methods. Aust. J. Phys. 41: 173-81

Macherauch E. Wohlfahrt H, Wolfsteig U 1973 Zur Zweckmassigen Definition von Eigenspannungen. Haerterei-Tech. Mitt. 28: 201-211

Nandi R K. Sengupta S P 1978 Analysis of X-ray diffraction profiles from imperfect solids by an application of convolution relations. $J$. Appl. Crystallogr. 11:6 $\div 9$

Pease R S 1948 The resolution of X-ray double diffraction lines into $\alpha_{1} \alpha_{2}$ components. J. Sci. Instrum. 5: 353

Rachinger W A 1948 A correction for $\alpha_{1} x_{2}$ doublet in the measurement of widths of X-ray diffraction lines. J. Sci. Instrum. 25: 254

Ramarao P, Anantharaman T R 1963 X-ray line breadth analysis of deformed metals. Z. Metallkd. 54: $658-663$

Rietveld H M 1967 Line profiles of neutron powder diffraction peaks for structure refinement. Acta Crystallogr. 22: $151 \quad 152$

Rietveld H M 1969 A profile refinement method for nuclear and magnetic structures. J. Appl. Crystallogr. 2: 65 - 71

Snyder R L 1993 The Rietveld method (ed.) R A Young (London: Oxford University Press) pp. $111 \cdot 131$

Stokes A R 1948 A numerical Fourier analysis method for the correction of widths and shapes of lines on X-ray powder photographs. Proc. Phys. Soc. London 61: 382 391

Suortti $\mathrm{P}$, Ahtee M, Unonious Z 1979 Voigt function fit of X-ray and neutron powder diffraction profiles. J. Appl. Crystallogr. 12: 365369

Taylor A $1961 X$-ray metallography (New York: John Wiley \& Sons) p. 686

Thompson P. Cox D E, Hastigs J B 1987 Rietveld refinement of Debye-Scherrer synchrotron $\mathrm{X}$-ray data from $\mathrm{Al}_{2} \mathrm{O}_{3}$.J. Appl. Crystallogr. 20:79 83

Toraya $\mathrm{H} 1986$ Whole powder pattern fitting without reference to a structure model: Application to X-ray powder diffractometer data. J. Appl, Crystallogr. 19:440 447

Treuting R G, Read W F Jr 1951 A mechanical determination of biaxial residual stress in sheet materials. J. Appl. Phys. 22: $130-134$ 
Warren B E $1969 X$-ray diffraction (Reading, MA: Addison-Wesley)

Warren B E, Averbach B L 1950 The effect of cold work distortion on X-ray patterns. J. Appl. Phys. 21: 595-599

Warren B E, Averbach B L 1952 The separation of cold work distortion and particle size broadening in X-ray patterns. J. Appl. Phys. 23: 497-498

Wilson A J C 1963 Mathematical theory of $X$-ray diffractometry (Eindhoven: Centrex)

Young R A 1980 Accuracy on powder diffraction. Natl. Bur. Stand. Special Publication 567 (eds) S Block, C R Hubbard (Washington, DC: NBS) pp 143-163

Young R A, Prince E, Sparks R A 1982 Suggested guidelines for the publication of Rietveld analyses and pattern decomposition studies. J. Appl. Crystallogr. 15: 357-358 